\title{
Morphological, Molecular Identification of Tabanidae (Diptera) and Assessment of their Seasonal Abundance in Southwestern, Saudi Arabia
}

\author{
N. I. Elobaid ${ }^{\text {* }}$, O. M. Daffalla ${ }^{2}$, S. S. M. Elhasan ${ }^{3}$, E. M. Noureldin², \\ F. F. Ali ${ }^{3}$ and M. A. Abdalla ${ }^{1}$ \\ ${ }^{1}$ Department of Preventive Medicine and Public Health, College of Veterinary medicine, \\ Sudan University of Science and Technology \\ ${ }^{2}$ Saudi National Centre for Disease Prevention and Control (SCDC) - Jazan, Saudi Arabia \\ ${ }^{3}$ Central Veterinary Research Laboratory (CVRL)- Khartoum, Sudan \\ *Corresponding author
}

Keywords

Tabanus, Chrysops, Haematopota, and Stomoxys

Article Info

Accepted:

12 March 2021

Available Online:

10 April 2021

\section{A B S T R A C T}

Tabanidae are true flies and members of the insect order Diptera, causing serious nuisance to livestock, which leads to considerable production losses and mechanically transmit several significant animal pathogens. In lack of data of classification and/or identification of tabanids (horse flies), additional to their seasonal abundance in the kingdom of Saudi Arabia (KSA), the present study was carried out to describe the morphological and molecular characterization of tabanid flies with density variation related. Four times a month, from October 2017 to September 2018, a total number of 457 flies' samples were collected from Jazan region southwestern Saudi Arabia, using Nzi traps. More tabanids flies were captured during the wet $67.61 \%$ (309/457) compared to the dry season $32.39 \%$ (148/457). Four different species of three Tabanids' genera were identified morphologically using the standard taxonomic keys; two for the first time namely; Hybomitra lurida and Tabanus par, beside Atylotus agrestis and Tabanus Sufis that were reported before in KSA. Genomic DNA was extracted from Saudi Arabian tabanids, and polymerase chain reaction (PCR) successfully amplified 710 base pairs of mitochondrial cytochrome c oxidase subunit 1 (COI) genes. Sequence analysis was performed, and three out of four species were identified ( $A$. agrestisMW243943, H. lurida MW265638 and T. parMW238412).The product sequences of KSA tabanid flies were aligned each separately against the corresponding GenBank sequences of known specimens and showed that the specimens of A. agrestis and T. par from Saudi Arabia were closely related to that of the Afrotropical region than to the Palaearctic region, whilst the sp. H. lurida was more similar to that of the Nearctic regions. This is the first report to investigate genetic characterization and phylogenesis of tabanid flies circulating in KSA. 


\section{Introduction}

On a world-wide scale, tabanids (horse flies) are among the major livestock pests, but the relative importance of most species varies temporally and geographically (1).Horse flies are poorly known in the Middle East (2), however, 31 species of the tabanids fauna have been identified and recorded in Saudi Arabia (3). Tabanidae family contains approximately 4455 described species (4), yet of these species have veterinary importance as significant pests of livestock, causing reductions in weight gain, milk yield and feedutilization efficiencies, due to their painful, continuous biting behavior (5). The bites of tabanids are painful, causing extreme annoyance and blood loss (up to $0.5 \mathrm{ml}$ per fly), with more blood loss following the bite due to oozing, local reactions to bites include dermal nodules and wounds which may serve as secondary feeding sites for other flies or as oviposition sites for myiasis-producing flies (1). Tabanids are mechanical vectors of over 35 pathogenic agents of livestock, including equine infectious anaemia virus, anaplasma marginale, Trypanosoma evansi and Trypanosoma vivax, as well as the agents of cutaneous anthrax, tularemia, bovine viral leukosis, vesicular stomatitis and hog cholera (6).Most people associate trypanosomosis with tsetse flies (Glossina spp), but tabanids play the most important role in the prevalence of the disease horizontally (transfer from one host to another). Generally, livestock suffer from trypanosomosis that is transmitted mechanically by several genera of hematophagous flies such as Tabanus, Chrysops, Haematopota and Stomoxys which are endemic in Africa, Asia and South America (7). Immediate mechanical transmission can only occur in a group of animals (e.g., intra herd transmission) and leads to a high incidence of disease in a given herd, however, it may occur between herds of the same species (camels) or of different species (camels and goats, e.g.) at a water point (8). The morphological keys and literature listed by Oldroyd (9) and Yagi (10), have been considered the main keys of identification of tabanids up to date. Nevertheless, a recent method of molecular DNA barcoding approaches based on the mitochondrial Cytochrome c Oxidase subunit 1 (COI) has been increased in popularity worldwide (11). Gene sequences have been used to discover new or previously unknown bio-diversities due to its ability to differentiate diverse arthropod species including insect pests. More recently, Morita (12) successfully revised the phylogenetic framework of Tabanidae by employing four sets of genes including the mitochondrial $\mathrm{COI}$ on a data set of 110 horsefly species (13).Control of horseflies is extremely difficult (14), but it can be attempted using trapping methods and/or impregnated screens or using insecticides on livestock. The accurate identification of tabanids species achieves detection of their victoria capacities to disease transmission, as well as tailoring their best surveillance and control program. Despite the fact that studies on vector ecology are essential for monitoring, understanding, predicting and controlling vector borne diseases, relatively few studies have been conducted on tabanids in Saudi Arabia and the Middle East as a whole. The aim of this study was to identify the tabanids species morphologically using taxonomic keys fauna complemented by molecular analysis based on (COI) barcoding as well as assessing their seasonal abundance in different habitats of Jazan region, southwestern Saudi Arabia.

\section{Materials and Methods}

\section{Study area}

The present study was conducted in 9 governorates of Jazan region (Jazan, Samttah, Abuareesh, Sabyah, Beash, Al-Darb, AlAridah, Al-Ahad and Al-Khobah). Jazan 
region, occupies an area of approximately $40,457 \mathrm{~km}$, is located in the southwest of Saudi Arabia between $16^{\circ}-12$, and $18^{\circ}-25$, latitude north. Bordered on the north and east by the Asir region and the Red Sea on the west along the coastline about $260 \mathrm{~km}$, whereas the Republic of Yemen from the south and southeast. The human population is estimated at about 1,603,659 (15).

The region includes more than 3500 villages and over 100 islands located in the Red Sea. (Fig.1), the geographic features are arranged in deserts, mountain ranges and open-low plains (less than 400 meters above sea level).

It is drained by several permanent valleys which play a crucial role in providing the perennial breeding habitats for insects, and several intermittent valleys (16). The Jazan region is situated in the subtropical zone and has average monthly temperatures ranging from $25.8^{\circ} \mathrm{C}$ in January to $33.4^{\circ} \mathrm{C}$ in July. The average relative humidity ranges between $55 \%$ and $72.5 \%$ (17). Annual rainfalls vary according to the area, in the plains, it ranges between 200- 500m min August and falling again in the months of March and April, while in mountains average from 529 to $630 \mathrm{~mm}$ (www.jazan.gov.sa). The vicinity of the region is considered to be a border point to the Yemen state and African Horne, in addition to the seaport, which plays a vital role in livestock's movement in and out of the country, and hence, provides the possibility of transmission of many infectious diseases.

\section{Samples Collection}

A total of 457 adult specimens of the family Tabanidae (Diptera) were collected from four distinct habitats of Jazan region during the period from October 2017 to September 2018. Tabanids collection was conducted from sunrise to sunset for four consecutive days a month, using Nzi traps (18). The traps were installed close to livestock, mostly camels.
The flies were collected daily after being killed naturally by insolation in plastic bottles and sorted by separating the tabanids from the other Diptera, then kept frozen until they were transferred to the laboratory for identification and enumeration.

\section{Morphological identification of field collected tabanids}

Classification of collected tabanids was achieved using standard taxonomic keys that differentiate between species as described by Oldroyd (9).

\section{Molecular description of preserved flies}

PCR targeting (COI) gene was performed to complement the morphological identification of tabanid flies and determine their phylogenetic position in comparison to other related taxa.

\section{Samples preparation and DNA extraction}

From each group of morphologically identical specimens, Legs of the insect samples were homogenized individually in a mortar and pestle (mini borosilicate glass chamber length $60 \mathrm{~mm} /$ pestle diameter $9.0 \mathrm{~mm}$ 3.0Ml, Fisher brand) in $100 \mu \mathrm{L}$ of Minimum Essential Media (MEM) (manufactured Euro Clone, UK). DNA was extracted from the stored homogenate using GeneJET Genomic DNA Purification Kit (Thermo Scientific) following the manufacturer's recommendations. The extracted DNA was stored at $-86^{\circ} \mathrm{C}$ until the next procedure.

\section{PCR amplification of tabanid flies DNA}

A pair of universal primers, LCOI490 (5GGTCAACAAAT CATAAAGATATTGG-3) and HCO2198 (5-TAAACTTCAG GGTGA CCAAAAAATCA-3) (19) were used to amplify the standard COI-5 barcodes (i.e. c. 650-bp fragments at the 5-terminus of the 
mitochondrial gene for COI; Folmer standardized region).

The PCR was carried out in total volume of 25 $\mu \mathrm{L}$ containing $12.5 \mu \mathrm{L}$ of $\mathrm{GoTag} \circledast \mathrm{G} 2$ green master mix ready to use from Promega, $2 \mu \mathrm{L}$ $(20 \mathrm{pmol})$ of each forward and reverse primers, $2 \mu \mathrm{L}$ of DNA template and $8.5 \mu \mathrm{L}$ nuclease free water. The mix was subjected to initial denaturation at $94^{\circ} \mathrm{C}$ for 3 minutes, 30 cycles of denaturation $\left(94^{\circ} \mathrm{C}, 60 \mathrm{~s}\right)$, primer annealing $\left(50^{\circ} \mathrm{C}, 60 \mathrm{~s}\right.$, primer extension $\left(72^{\circ} \mathrm{C}, 60 \mathrm{~s}\right)$ and final extension for 5 minutes. In each run, negative and positive controls were included.

The PCR products amplification was analyzed by gel electrophoresis (1.5 Agarose in TrisAcetate EDTA buffer) staining with ethidium bromide. The visualization was carried out using Gel Doc XR Imaging System (Bio-Rad).

\section{Sequencing of PCR Fragment}

Purification and standard sequencing for COIPCR products were performed by Macrogen Company (Seoul, Korea). Sequencing reactions were performed in a MJ Research PTC-225 Peltier Thermal Cycler using a ABI PRISM ${ }^{\circledR} \quad$ BigDye $^{\mathrm{TM}}$ Terminator Cycle Sequencing Kits with AmpliTaq ${ }^{\circledR}$ DNA polymerase (FS enzyme) (Applied Biosystems), following the protocols supplied by the manufacturer. Single-pass sequencing was performed on each template using D1 (forward) primer.

The fluorescent-labeled fragments were purified from the unincorporated terminators with BigDye ${ }^{\circledR X}$ Terminator ${ }^{\mathrm{TM}}$ purification protocol. The samples were resuspended in distilled water and subjected to electrophoresis in an ABI 3730xl sequencer (Applied Biosystems).

\section{Bioinformatics and sequence analysis}

The sequences were searched for genetic similarity through Basic Local Alignment
Search Tool (BLAST) data (www.ncbi.nlm.nih.gov/BLAST/) (20) and compared to reference sequences of tabanids isolates detected in BLAST and downloaded from Gen Bank (www.ncbi.nlm.nih.gov /genbank/).

\section{Phylogenetic analysis}

Multiple sequence alignments for COI-PCR products were conducted using bioEdit table, version A (21). Phylogenetic tree of COI-PCR products of nucleotide sequences was run using neighbor-joining algorithms by Mega7 programme (http://www.megasoftware.net/) (22). The evolutionary history was inferred using the Neighbor-Joining method (23). The tree is drawn to scale, with branch lengths in the same units as those of the evolutionary distances used to infer the phylogenetic tree, the evolutionary distances were computed using the Maximum Composite Likelihood method (24) and is in the units of the number of base substitutions per site.

\section{Results and Discussion}

\section{Morphological identification of tabanids collected}

A total of 457 adult specimens of the family Tabanidae (Diptera) were collected from four distinct habitats of the Jazan region. The morphological classification revealed a total of three different genera namely:Tabanus (91.03\%), Atylotus $(7.88 \%)$ and Hybomitra $(1.09 \%)$ with a total of four different species $A$. agrestis, H. lurida, T. Sufis and T. par. We identified tabanid species based on their morphological features described by Oldroyd (9).

\section{Genus Atylotus Osten - Sacken, 1876}

\section{Description}

Small to medium sized horse flies, grayish, yellowish or yellowish-brown, characterized 
with large heads, bare or pubescent eyes, sometimes with one narrow band. Males have sharply separated areas of small facets from the area of large facets from the upper part of the eyes. Frontal calli in females are always very small, round and reduced, or sometimes absent. Wings are clear with a very long appendix to vein R4 (25).

\section{Atylotus agrestis Wiedemann, 1828}

A female of this specimen was identified by characteristics described by Oldroyd (9).Head: Eyes usually bare, rusty brown in dried specimens, with a trace of a single purple band usually visible; in life specimens they are very pale green with dark spots shining through. Upper and lower calliare circular, light brown, shining. Subcallusis flat; sometimes rises a little towards the antennae.

Palpi white, rather swollen at the base, drawn out into long points; hairs mostly white with many black ones intermingled. Propose orange. Antennae light orange - yellow, first segment with thick whitish tomentum.

Thorax: mesonotum black in ground colour, covered with brownish grey tomentum; very slight traces of four brown longitudinal strips. Scutellumis uniformly brownish. Abdomen: in ground colour ashy grey-brown, with two sublateral yellowish or orange stripes. Legs: Coxey likes pleura, except that the fore pair is yellowish. Femora are yellowish. Fore tibiae: light brown Fore tarsi: dark brown Middle tarsi: brown. The rests of the legs are all reddish yellow. Wings: Veins, including costa, pale yellow, only stigma slightly brownish. Length: Body $14 \mathrm{~mm}$; Wing $11 \mathrm{~mm}$.

\section{Genus Hybomitra Enderlein, 1922}

\section{Description}

Medium-sized to large species, thorax and abdomen always densely pubescent. Most species have yellowish-brown margin on the abdomen or grayish-black to completely black species. Eyes are greenish, always pubescent, usually with three bands, very rarely unbanded or with one or two bands. Ocellar tubercles are always present in both sexes. Wings are clear, slightly pale brown clouded at the base of the wing (26).

\section{Hybomitra lurida Fallén1817}

A female of this specimen was identified by characteristics described by Oldroyd (9). Subcallus partly bare and shining; head strongly transverse, lower angle between eyes greater than $90^{\circ}$ (view head face-on). In hind view, tergites with broad median stripes remain shining black, together with some more lateral areas.

\section{Genus Tabanus Linnaeus, 1758}

\section{Description}

Medium-sized to large, mostly are black to blackish-gray, or chestnut brown species. Abdomen has pale median triangles and sublateral patches, or more or less distinct brown side markings. Eyes are bare or pubescent, without bands or with one to four bands. Females with well developed, large frontal calli specifically shaped and positioned. Fron's size varies from broad to very narrow, without ocellar swelling.

Wings are mostly clear. Antennae consist of more or less distinct dorsal teeth to 3rd antennal segments and the terminal part of the flagellum form of four segments. Basicosta covered with short hairs (25).

\section{Tabanus Sufis Jaennicke, 1867}

A female of this specimen was identified by characteristics described by Oldroyd (9).Head: Eyes microscopically hairy; in life bright green with four horizontal bands, the middle 
two deep blue and the others red-brown, the lowest one being on the lower margin of the eye. Frons is very strongly convergent. Calli yellow: both upper and lower callus transverse, with an irregular margin, and extending across the frons from eye to eye; the two calli are separated in fresh specimens, but are sometimes joined by rubbing. Subcallus flat, on the upper margin is a divided area, shining yellowish. Palpi plump, tapering sharply to a point, clear with white hairs. Antennae are entirely orange. Thorax: mesonotum ashy grey with three continuous, narrow longitudinal stripes of pale grey; scutellum ashy grey. Abdomen: Dorsum dark brown with grey hindmargins and side margins, with three rows of grey spots: median spots triangular, extending to front margin of each segment; sublateral spots, on all segments. The rows of sublateral spots don't unite into a stripe. Legs: Coxey like pleura. Femora are yellowish grey with white hairs. Tibiae and tarsi are yellowish. Wings: Quite clear, with stigma almost invisible and with the veins colorless. Vein $\mathrm{R}_{4}$ is a welldeveloped appendix. Length: Body $8-11 \mathrm{~mm}$; Wing $8-10 \mathrm{~mm}$.

\section{Tabanus par Walker, 1858}

A female of this specimen was identified by characteristics described by Oldroyd (9). Head: Eyes in life are brilliant emerald green, without bands. Frons is convergent. Calli is clear yellow and broadly united into an elongate, onion -shape. Subcallus often denuded. Palpi yellow, with some black hairs. Proboscis is yellow or orange. Antennae: first two segments yellow with yellow hairs. Thorax: mesonotum and scutellum black in ground colour, with a uniform covering of yellow-grey tomentum. Pleura grey or a little yellowish, with long white hairs. Abdomen:
Dorsum orange, without pattern. Venter is more whitish, with very pale yellow hairs. Legs: Coxey like pleuragreys with whitish hairs. Legs are otherwise yellow to reddish brown, tarsi a deeper brown than rest. Hairs femora yellowish, those of tibiae mixed with black ones. Wings:Quite clear veins and stigma yellow. Length: Body 12 mm; Wing 11 $\mathrm{mm}$.

\section{Molecular characterization of collected flies}

A partial sequencing was done for twenty five PCR-COI product samples $(710 \mathrm{bp})$ representing the specimens of collected tabanid flies to supplement the morphological identification. The sequences analysis generated only three species of isolates, were named in three terms of; Saudi Arabia $A$. agrestis, Saudi Arabia H. lurida and Saudi Arabia T. par, released in GenBank with accession numbers; MW243943, MW265638 andMW238412, respectively. BLAST data showed different identical between the sequences of Saudi Arabia isolates and other sequences of tabanids selected globally from GenBank, for KSA isolates of A. agrestis sequence produced the $92-99 \%$ similarity with other tabanids isolate from Mozambique MT231182, Senegal MW013785, China KT225291, USA KM243503and Thailand MG426044. BLAST for KSA isolates of $H$. lurida sequence showed the 92-94\% similarity with other tabanids isolates such as USA KU875227, Canada JF875803, Denmark MT410834, Thailand MG426176 and Myanmar MF804680. BLAST for KSA isolates of $T$. par sequence produced the 9192.80\% similarity with other tabanids isolates from Central African Republic (CAR) MK396308 India KM111678 Gabon MK396326, ThailandMG426116, and USA KM243535. 
Table.1 Density variation of Tabanids flies collected among seasons of the year of current study.

\begin{tabular}{|c|c|c|c|c|c|c|c|c|}
\hline \multirow{2}{*}{$\begin{array}{c}\text { Season of } \\
\text { collection }\end{array}$} & \multicolumn{2}{|c|}{ Tabanus sp. } & \multicolumn{2}{c|}{ Atylotus sp. } & \multicolumn{2}{c|}{ Hybomitra sp. } & \multicolumn{2}{c|}{ Total } \\
\cline { 2 - 9 } & $\begin{array}{c}\text { Collected } \\
\text { Number }\end{array}$ & $\%$ & $\begin{array}{c}\text { Collected } \\
\text { Number }\end{array}$ & $\%$ & $\begin{array}{c}\text { Collected } \\
\text { Number }\end{array}$ & $\%$ & $\begin{array}{c}\text { Collected } \\
\text { Number }\end{array}$ & $\%$ \\
\hline Wet season & 278 & 89.97 & 29 & 9.39 & 2 & 0.65 & 309 & 67.61 \\
\hline Dry season & 138 & 93.24 & 7 & 4.73 & 3 & 2.03 & 148 & 32.39 \\
\hline Total & 416 & 91.03 & 36 & 7.88 & 5 & 1.09 & 457 & 100 \\
\hline
\end{tabular}

Fig.1 Map of Jazan region showing different governorates.

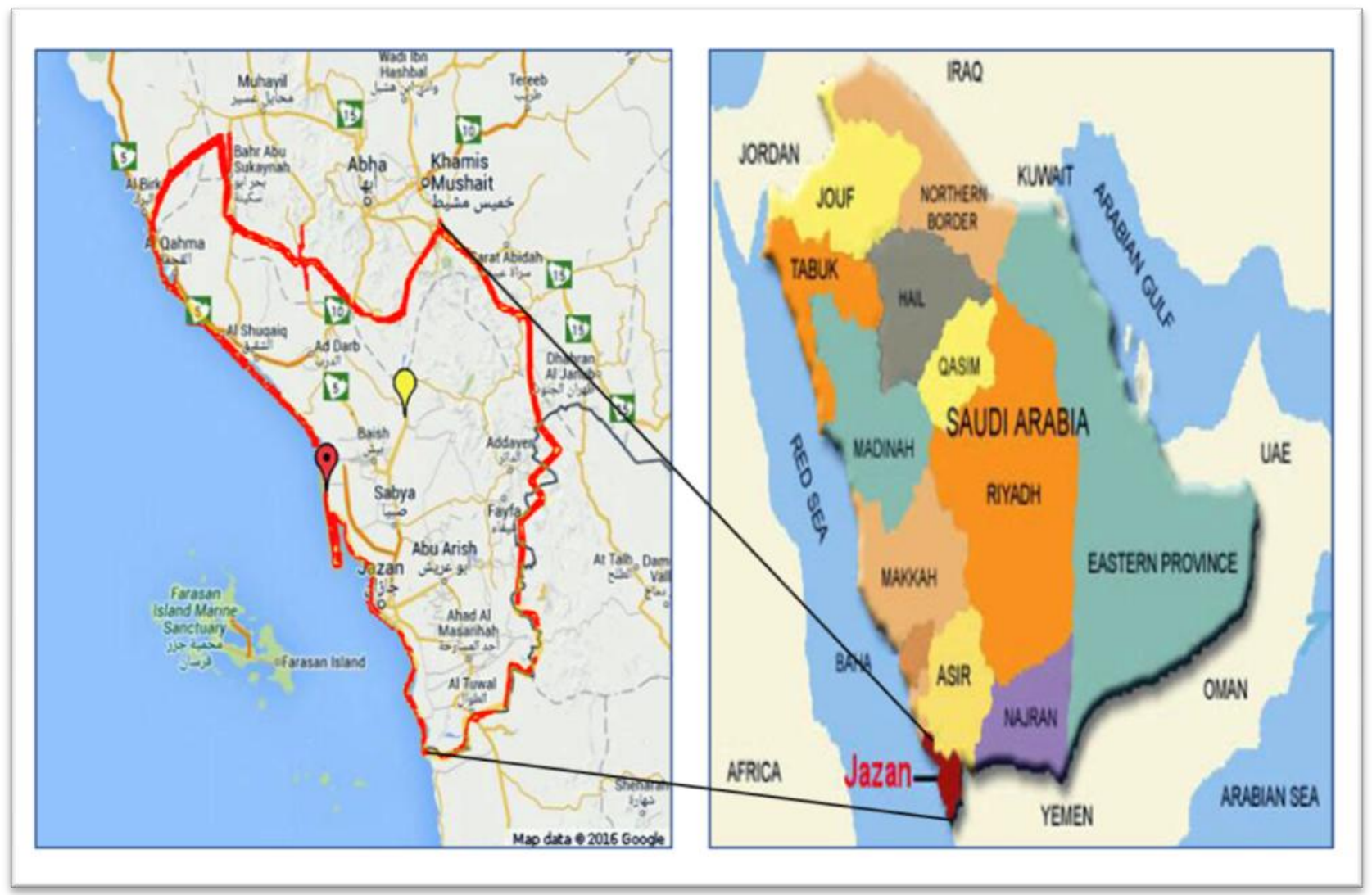


Fig.2 Multiple sequence alignment of COI gene for cytochrome oxidase subunit 1 of KSAT. par. Identical bases are shown as dots and sites of variation are shown.

SaudiArabia_T.par
Central African Republic MR396
Gabon MK396328
India KM111678
India KM111672
Gabon MR396326
Thailand MG426116
Liberia MIR396356
USA RM243535
USA RM243541
Mozambique MT231163

SaudiArabia T.par Central African Republic MrK396 Gabon MIK396328 India RM111678 India RM111672 Gabon MK396326 Thailand MG426116 Liberia MIK396356 USA KM243535 USA KM243541

Mozambique MT231163

SaudiArabia T.par Central African Republic MR396 Gabon MIK396328 ndia RM111678

India RM111672

Gabon MIK396326

Thailand MG426116

Liberia MK396356

USA KM2 43535

USA RMI243541

Mozambique MT231163

SaudiArabia_T.par Central African Republic MIK396 Gabon MK396328 India $\mathrm{KM} 111678$

India RM111672

Gabon MK396326

Thailand MG426116

Liberia MR396356

USA RM243535

USA RMN243541

Mozambique MT231163

SaudiArabia T.par

Central African Republic MIK396 Gabon MIK396328

India KM111678

India KM111672

Gabon MIR396326

Thailand MG426116

Liberia MIK396356

USA RM243535

USA RM243541

Mozambique MT231163

SaudiArabia T.par

Central African Republic MIK396 Gabon MIK396328

India KM111678

India RM111672

Gabon MIK396326

Thailand MG426116

Liberia MIR396356

USA KM2 43535

USA RM243541

Mozambique MT231163

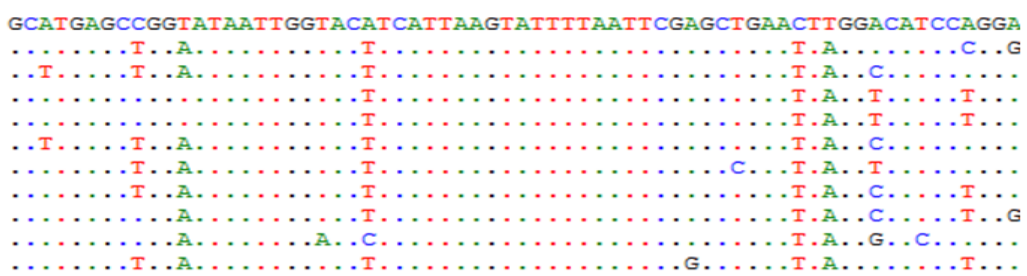

121 GTIATAATTITCITTATAGTAATACCTATTATAATTGGAGGATTTGGAAATTGATTAGTICCTITA

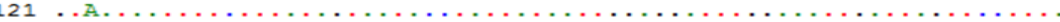

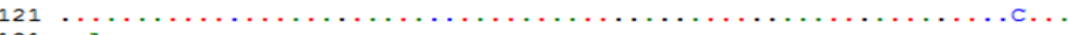

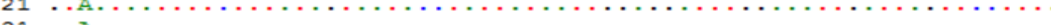

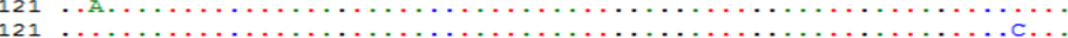
$121 \quad \cdots \cdots \cdots \cdots \cdots \cdots \cdots \cdots \cdots \cdots \cdots \cdots \cdots$

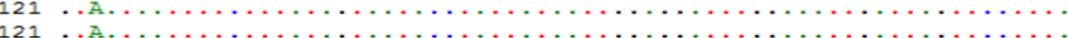

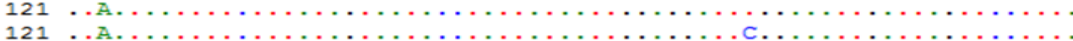

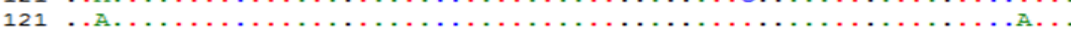
$121 \ldots$ A. . . .

241 ITATTACCTCCATCATTAACTCTTTIATTAGCTAGTAGTATAGTAGAAAATGGAGCTGGGACTGGI

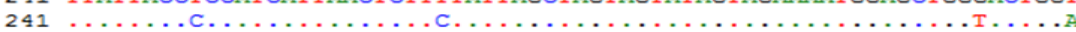

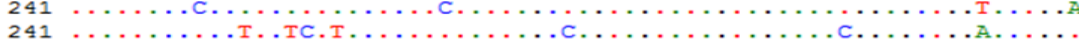

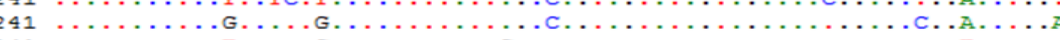

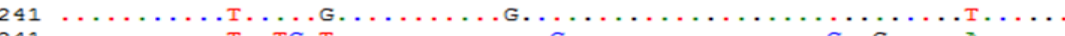
$241 \ldots$. . . . . . . . . . . . . . . . . . . . . . . . . .

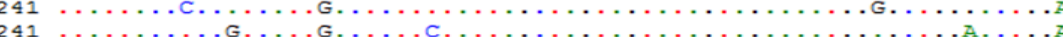

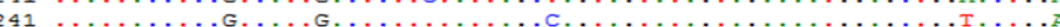

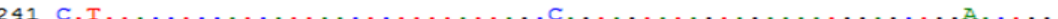
241 …

361 GATTTAGCAATTTTTICTCTTCATTTAGCAGGAATTTCTTCTATTCTAGGAGCTGTAAACTTTATI $361 \ldots \ldots \ldots$. . . . . . . . . . . . . . . . . . . . . . . . .

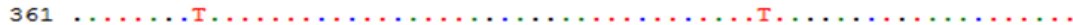

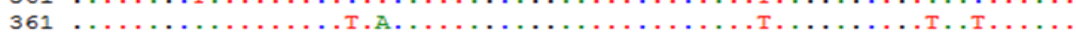

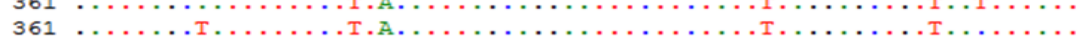

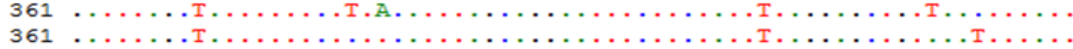

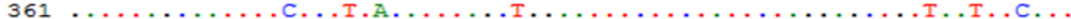

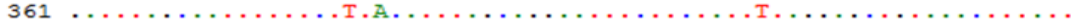

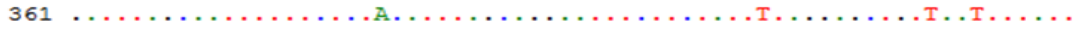
$361 \ldots \ldots \ldots \ldots \ldots \ldots \ldots \ldots \ldots \ldots \ldots \ldots \ldots \ldots \ldots \ldots$.. A. . . . . . . . . . $361 \ldots \ldots$. . . . . . . . . . . . . . . . . . .

481 TTIGTTIGAGCAGTAGTTATTACTGCTATTTTACTTTTACTTTCATTACCTGTATTAGCTGGAGCI

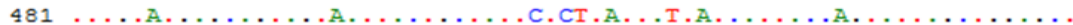
$481 \ldots \ldots \ldots$. . . . . . . . . . . . . . . . . . . . . .

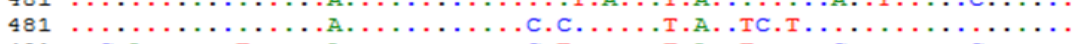
481 . С.А. . . . . . . . . . . . . . . . . . . . . . . . . . . $481 \ldots \ldots \ldots \ldots \ldots$. . . . . . . . . . . . . . . . . . . . . $481 \ldots \ldots \ldots \ldots \ldots$. . . . . . . . . . . . . . . . . . . . . $481 \ldots$. . . . . . A. . . . . . . . . . . . . . . . . . . . . $481 \ldots \ldots \ldots$. $\ldots \ldots$. . . . . . . . . . . . . . . . . .

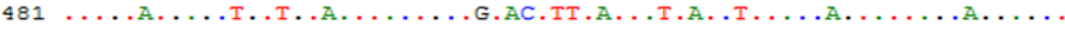
$481 \ldots$. . . . . . . . . . . . . . . . . . . . . . . . . . .

601 GGAGGTGGAGATCCTATTTTATACCAACATTTATTT

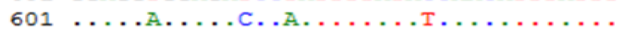

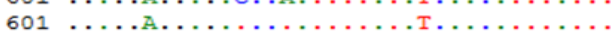

601 … А. . . .

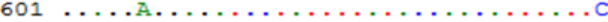

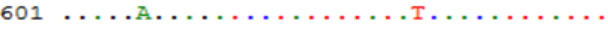

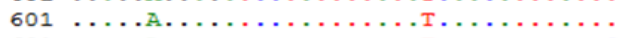

$601 \ldots \ldots \ldots \ldots \ldots \ldots$. $\ldots \ldots \ldots \ldots \ldots$.

$601 \ldots$. . . . . . . . . . . . . . . .

$601 \ldots \ldots$. $\ldots \ldots \ldots \ldots \ldots$. $\ldots \ldots \ldots \ldots \ldots$

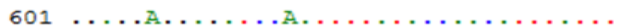


Fig.3 Multiple sequence alignment of COI gene for cytochrome oxidase subunit 1 of KSAA. agrestis. Identical bases are shown as dots and sites of variation are shown.

SaudiArabia_Atylotus agrestis
MozambiqueMT231182
Kenya KX946514
Kenya KX946508
Senegal MW013785
South Korea KC136014
Canada JF869012
Canada KM570795
USA RM243503
Thailand MG426044

SandiArabia Atylotus agrestis Mozambi queMT'231182

Kenya KX946514

Kenya KX946508

Senegal Mw013785

South Korea KC136014

Canada JF869012

Canada KM570795

USA RM1243503

Thailand MG426044

SaudiArabia_Atylotus agrestis

MozambiqueMT'231182

Kenya KX946514

Kenya KX946508

Senegal MW013785

South Korea KC136014

Canada JF869012

Canada KM570795

USA RM243503

Thailand MG426044

SaudiArabia Atylotus agrestis Mozambi queMT'231182

Kenya KX946514

Kenya KX946508

Senegal Mw013785

South Korea KC136014

Canada JF869012

Canada RM570795

USA RM243503

Thailand MG426044

SaudiArabia_Atylotus agrestis

Mozambi queMT231182

Kenya KX946514

Kenya KX946508

Senegal Mw013785

South Korea KC136014

Canada JF869012

Canada RM570795

USA RM243503

Thailand MG426044

SaudiArabia_Atylotus agrestis MozambiqueMT231182

Kenya KX946514

Kenya KX946508

Senegal MW013785

South Korea KC136014

Canada JF869012

Canada RM570795

USA RM243503

Thailand MG426044

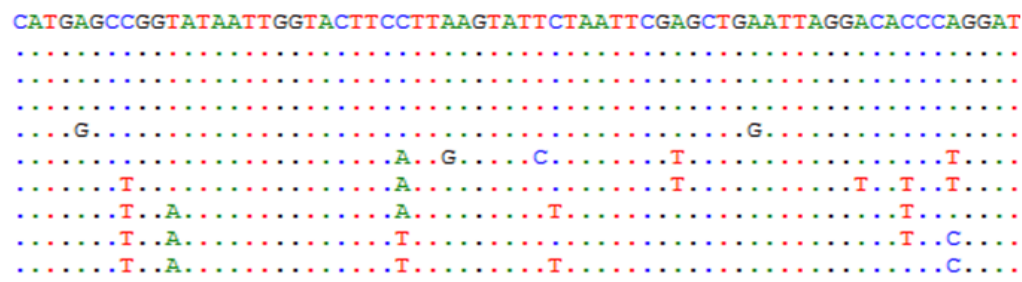

TGTAACTGCACATGCTTTTGTTATAATTTTCTTTATAGTAATACCTATTATAATTGGAGGATTTGG

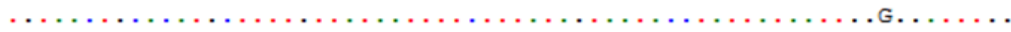

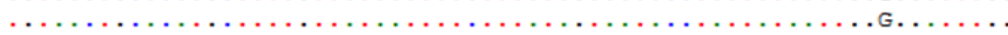

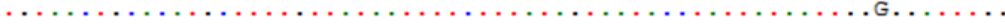

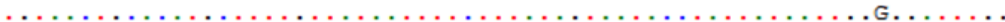

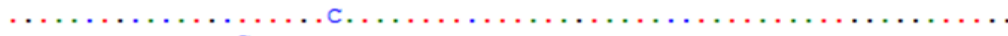

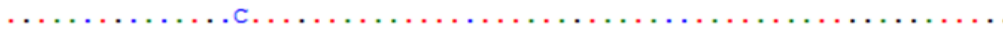

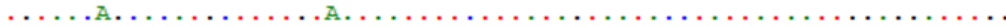

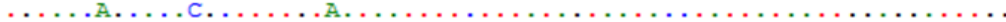

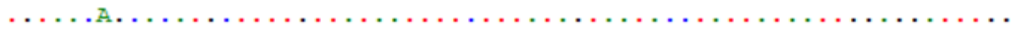

GATATAGCATTTCCTCGAATAAATAATATAAGTTTTTGATTACTACCTCCCTCATTAACCCTTTTA

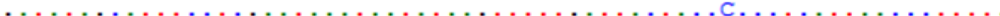

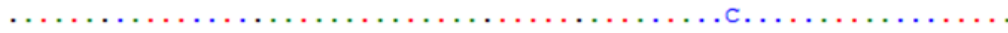

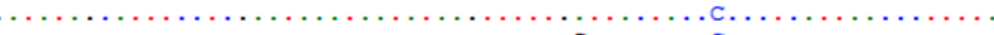

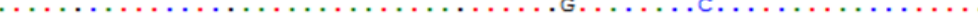

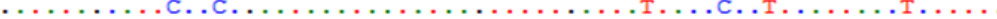

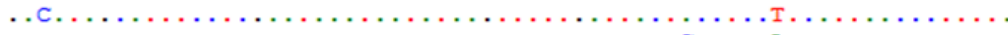

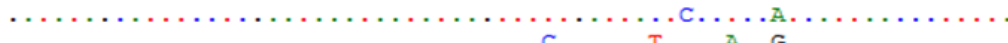

301 CAGGATGAACAGTTTATCCTCCTCTATCTGCTGCAATTGCTCATGGAGGAGGTTCTGTTGATTTAG

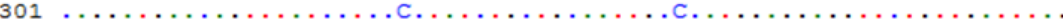

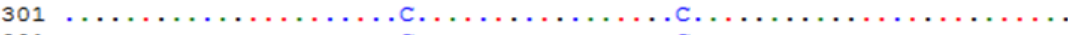

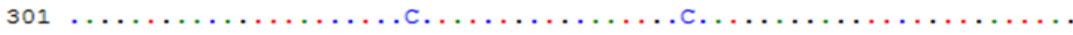

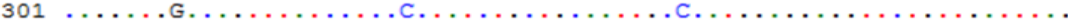
301 . $\ldots \ldots \ldots \ldots \ldots$. . . . . . . . . . . . . . . . . . . . 301. . . . . . . . . . . . . . . . . . . . . . . . . . . . 301. Т. . . . . . . . . . . . . . . . . . . .

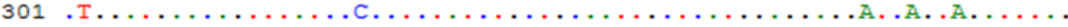

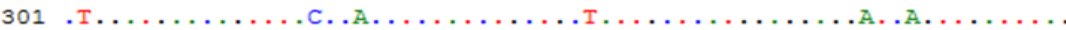

401 TATTTTAGGAGCTGTAAATTTTATTACAACAGTAATTAATATACGATCTACAGGAATTACCTTTGA 401

401

401

401

401

401

401

401

40

501

501

50

501

501

501

$501 \ldots$ Т.

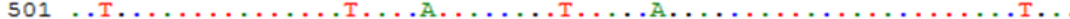


Fig.4 Multiple sequence alignment of COI gene for cytochrome oxidase subunit 1of KSAH. lurida. Identical bases are shown as dots and sites of variation are shown.

SaudiArabia_H. Lurida
USA KU875227
USA KU875226
Canada JF875803
Canada KJ089802
Canada KR643565
Canada KR661984
Canada KM570780
Denmark MT410834
Thailand MG426176
Myanmar MF804680

SaudiArabia H. Lurida USA KU875227

USA KU875226

Canada JF875803

Canada KJ089802

Canada KR643565

Canada KR661984

Canada KM570780

Denmark MT410834

Thailand MG426176

Myanmar MF804680

SaudiArabia H. Lurida USA KU875227

USA KU875226

Canada JF875803

Canada RJ089802

Canada KR643565

Canada KR661984 Canada KM570780

Denmark MT410834 Thailand MG426176

Myanmar MF804680

SaudiArabia H. Lurida USA KU875227

USA KU875226

Canada JF875803

Canada KJ089802

Canada KR643565

Canada KR661984

Canada KM570780

Denmark MT410834

Thailand MG426176

Myanmar MF804680

SaudiArabia_H.Lurida USA KU875227

USA KU875226

Canada JF875803

Canada KJ089802

Canada KR643565

Canada KR661984

Canada KM570780

Denmark MT410834

Thailand MG426176

Myanmar MF804680

SaudiArabia_H. Lurida USA KU875227

USA KU875226

Canada JF875803

Canada KJ089802

Canada KR643565

Canada KR661984

Canada KM570780

Denmark MT410834

Thailand MG426176

Myanmar MF804680

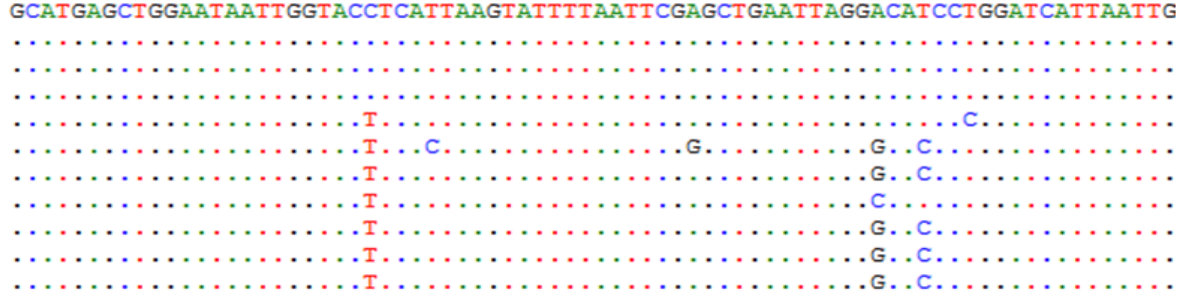

GTAATAATTTCCTTATAGTAATACCTATTATAATTGGAGGATTTGGAAATTGATTAGTTCCTTTAATATTAGGAG

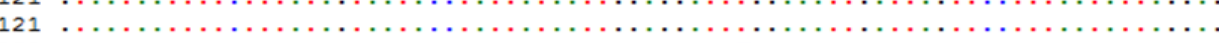

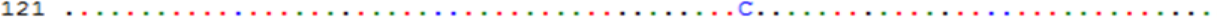

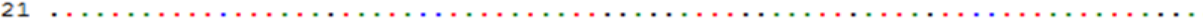

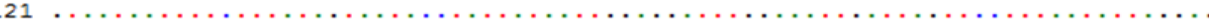

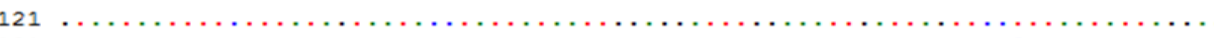

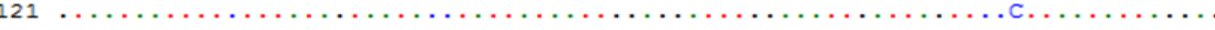

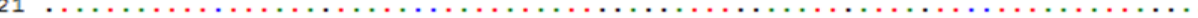
$121, \ldots$ 121

241 TTACTTCCACCATCACTGACTCTTTTATTAGCCAGTAGTATAGTAGAAAATGGGGCTGGAACTGGATGAACAGTTI

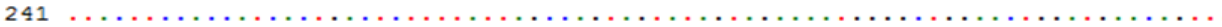

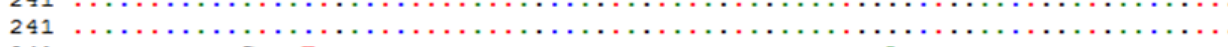

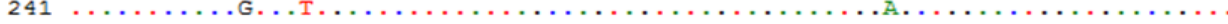

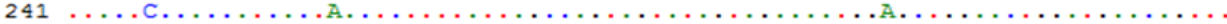

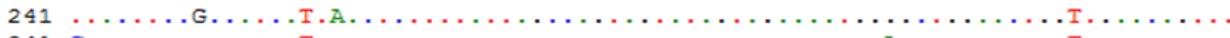

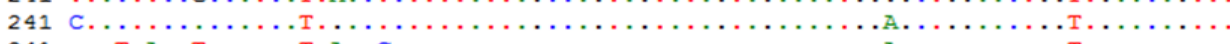

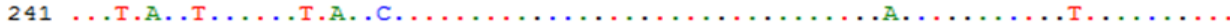

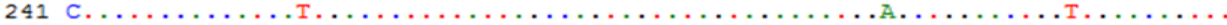

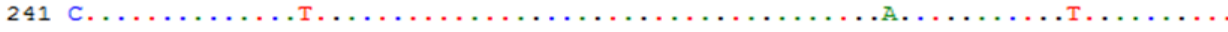

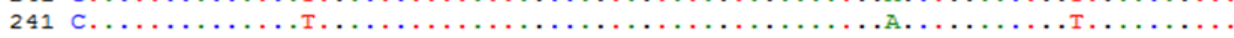

361 GATTTAGCAATTTTTICTCTACACTTAGCCGGAATTTATTCTATTTTAGGAGCTGTAAACTTTATTACTACTGTTA

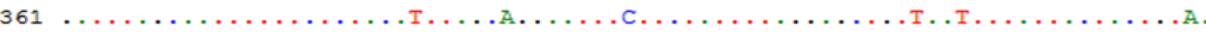

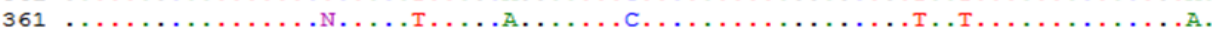
$361 \ldots \ldots \ldots \ldots \ldots$. $\ldots \ldots$. $\ldots$. . . . . . . . . . . . . . . . . . $361 \ldots \ldots \ldots \ldots \ldots \ldots$ I. . . . . . . . . . . . . . . . . . .

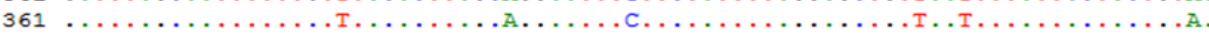
$361 \ldots \ldots \ldots \ldots \ldots$. $\ldots$ AT. . . . . . . . . . . . . . . . $361 \ldots \ldots \ldots \ldots \ldots$. . . . . . . . . . . . . . . . . . . . . . . . $361 \ldots \ldots \ldots \ldots \ldots$ AT . . . . . . . . . . . . . . . . . $361 \ldots \ldots \ldots \ldots \ldots$. $\ldots$. . . . . . . . . . . . . . .

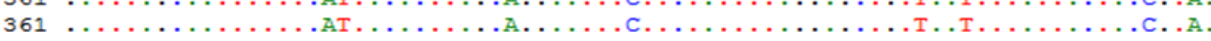

481 TTTGTTTGAGCTGTATTTATTACTGCTATTTTACTATTACTTTCATTACCAGCATTAACTGGAGCTATTACTATAT

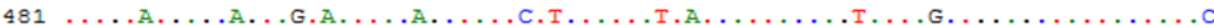

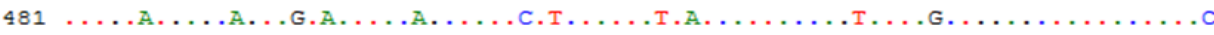

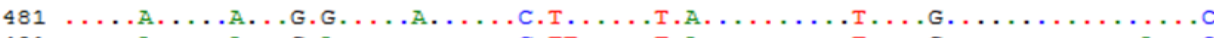
$481 \ldots$. . . . . . . . . . . . . . . . . . . . . . . . . . . . . . . .

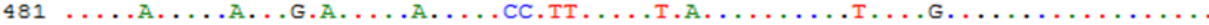

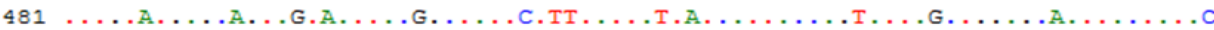
$481 \ldots$. . . . . . . . . . . . . . . . . . . . . . . . . . . . . . . . . . .

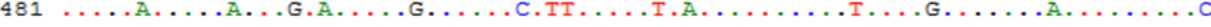

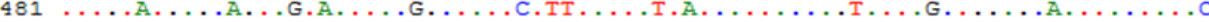

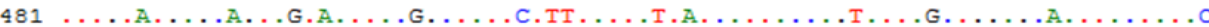

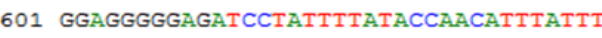

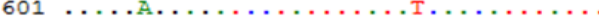

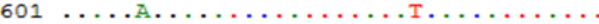

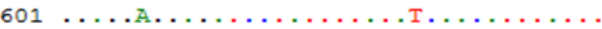

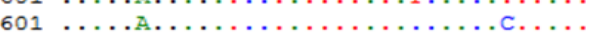

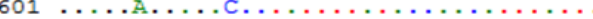

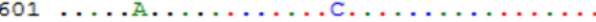

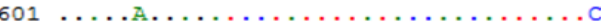

$601 \quad \ldots \ldots$ A. . . . . . . . . . . . . .

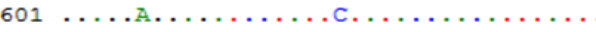

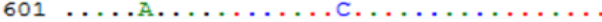


Fig.5 Phylogenetic tree based on the (COI) gene of KSA isolates of tabanid flies with other known different countries. The proportion of sites where at least 1 unambiguous base is present in at least 1 sequence for each descendent clade is shown next to each internal node in the tree.

The analysis involved 17 nucleotide sequences and all positions containing gaps and missing data were eliminated, there were a total of 634 positions in the final dataset.

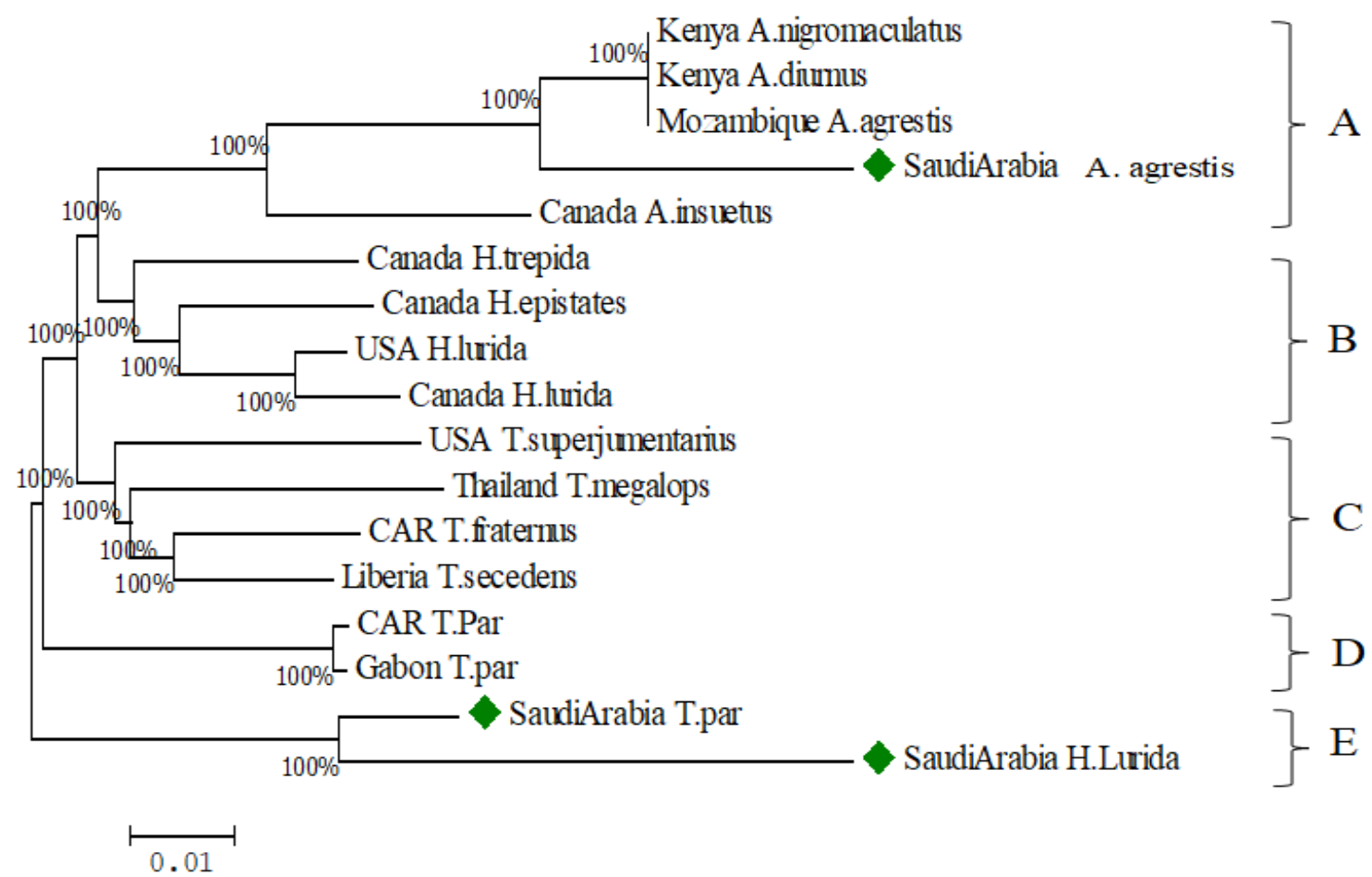

Phylogenetics of Saudi Arabian tabanid flies

A multiple sequence alignment of (COI) gene and molecular phylogenetic analysis were performed to demonstrate the relationship between the KSA isolates of tabanids each separately with the other global isolates (Figs.2-4), and were tightly clustered together in phylogenetic tree, as shown in Fig (5).

\section{Seasonal occurrence and relative abundance of tabanids collected}

To find out the seasonal abundance and density variation of collected tabanids, a number of 309 (T. par $=278$, A. agrestis $=29$ and $H$. laurida $=2$ ) were caught during wet, while 148 tabanids flies (T. par $=138, A$. agrestis $=7$ and $H$. laurida $=3$ ) were captured in dry season, as summarized in table (1).

Tabanid flies have a passive economic effect and are known worldwide as important mechanical vectors of viruses, bacteria, protozoans and helminths, which cause diseases in some wild and domestic animals $(27,28)$ in addition to reducing milk production where they commonly live (29). In Saudi Arabia, 31 species of tabanid flies have been identified and listed (3).In the current study the morphological identification revealed the presence of four species of tabanids flies, two for the first time namely; $H$. luridaand $T$. par, beside A. agrestis and $T$. sufis which were recorded previously in KSA. 
To confirm and complement the morphological results, a partial sequencing was done and amplified successfully for twenty five PCR specimens targeting 710 bp of mitochondrial Cytochrome $\mathrm{c}$ Oxidase subunit 1 (COI) gene of captured horse flies. The BLAST data searched for nucleotide sequences and confined only to three species of A. agrestis (MW243943), H. lurida (MW265638) and T. par (MW238412). Nucleotide sequence alignment of COI gene showed similarity between the specimens of $T$. par and A. agrestis from Saudi Arabia to that of the Afrotropical region than to the Palaearctic region, whilst $H$. lurida specimen was closely related to that of the Nearctic and the Neotropical regions. Yet, our findings of molecular analysis showed higher identity between Saudi Arabian tabanids to known specimens but differ in species, for instance KSA Atylotus agrestis (MW243943) corresponds to Atylotus nigromaculatus (KX946514) from Kenya, Atylotus miser (KT225291) from china, and Hybomitra bimaculata (KM243503) from USA. KSA Hybomitra lurida (MW265638) corresponds to Hybomitra epistates (KJ089802) from Canada, Hybomitra bimaculata (MT410834) from Denmark, and Tabanus tamthaiorum (MG426176) from Thailand. KSA Tabanus par (MW238412) corresponds to Tabanus striatus (KM111678) from India, Tabanus fraternus (MT231163) from Mozambique, and Tabanus secedens (MK396356) from Liberia. Thus, the need to revise the morphological taxonomy of tabanid flies worldwide is too necessary to be more accurate. The phylogenetic relationships among Saudi Arabian tabanids were inferred each separately and allied species were tightly clustered into a well-supported tabanids clade by analysis of targeting the (COI) gene, and were distinct genetically from all other global tabanids. The phylogenetic tree showed five major Clades (A-E), namely, clade A-Atylotus spp.; clade B- Hybomitra spp.; clade C, clade
D- Tabanus spp and clade E $-T$. par and $H$. lurida of KSA. It is worthy to note that the genera Atylotus, Hybomitra and Tabanus formed a paraphyletic clade with each other (Fig.5). Interestingly, T. par (MW238412) and $H$. lurida (MW265638) of KSA branched on own clade, this finding maybe explained with the results reported by Yeates (30) and Morita (12) who mentioned that in most phylogenetic analyses of tabanid flies and other closely related species there are normally uncertainties with the position of most species due to weak support observed from internal relationships. Among others, these studies strongly suggest that DNA barcoding is a promising tool for diversity studies, phylogeographic analyses as well as evolutionary studies of arthropods specifically as vectors, and thus could provide a globally important tool for controlling pests (13). The seasonal abundance patterns of tabanid flies in the study areas of present study showed that themajority of tabanid flies were collected during the wet $67.61 \%$ (October to February), compared to the dry season $32.39 \%$ (Mars to September), a result which is in line with the findings of Barros (28), Itina (31) and Baldacchino (32) who gained the high incidence of tabanids was in the wet compared to dry season. Temperature, relative humidity (RH) and precipitation are factors related either separately or interactively to fluctuate the abundance of insect populations. The temperature influences the rate of development of the immature stages and eggs production, $\mathrm{RH}$ interferes with survival rate and richness of adult flies (33), while it seems that the precipitation influenced tabanids population by the previous year's precipitation in Korea and Croatia (34,35). Issimov (36) reported that, the humidity and ambient temperature play a decisive role in the active formation and emerging of adult insects in Europe, and the most suitable climatic indicators were humidity at $70 \%$ and temperature of $27^{\circ} \mathrm{C}$ for adults, whilst the 
eggs hatch was highest at $32^{\circ} \mathrm{C}, \mathrm{RH}$ at $90 \%$ and lowest at $27^{\circ} \mathrm{C}$. Consequently, the number of horse fly species begin to increase in Europe at the second half of May (spring in the northern hemisphere) and decreases in the middle of September (late summer) $(29,32,37)$ in contrary of the occurrence rate of tabanids in tropical and subtropical zones, for example in Brazil, the tabanids population increase in September, coinciding with the beginning of the rainy season (28). A better coverage of the tabanids in different ecosystems, including the conservation areas and farming communities, will allow better understanding of the risk of transmission of diseases vectored by these flies in both wild and domestic animals (13). This is the first report that studies the molecular identifications of diverse tabanids from the kingdom of Saudi Arabia, as well as studying their seasonal abundance. The collected tabanids presented in this study are confined to specific area given the limited study site. Thus, further investigations are required to; 1 - Comprehend the whole fauna of tabanid flies and their habitats using large various methods of trapability with assessing the climatic factors influencing their development.2- Detect protozoan parasites they are harbouring using molecular technique and other related pathogens circulating in the region.3- Estimate the effect of human activity on their distribution and prevalence, in addition to enabling the accurate identification of tabanid flies will enhance and promote more efficient control measures against them as disease vectors.

\section{References}

Foil L. D and J. A. Hogsette, (1994). "Biology and control of tabanids, stable flies and horn flies" Rev. Sci. Tech. off. Int. Epiz., 1994, 13 (4), pp. 1125-1158.

Al-Talafha, H., Amr, Z. S., Abu Baker, M., Katbeh Bader, A., (2004). Systematics and distribution of horse flies (Diptera:
Tabanidae) of Jordan. Journal of Vector Ecology 30, 49-61.

Al-Dhafer. H. M, H. A. Dawah, M. A. Abdullah, (2009). Tabanidae (Diptera) of Saudi Arabia. Saudi Journal of Biological Sciences 16:77-83.

Pape, T., Blagoderov, V. \& Mostovski, M. B. (2011). Order Diptera Linnaeus, 1758. Animal Biodiversity: An Outline of Higher-level Classification and Survey of Taxonomic Richness (ed. by Z.-Q. Zhang). Magnolia Press, Auckland, New Zealand. pp. 222-229.

Croof I. M. N. Hamid, Mai M. Nour, Nahla O. M. Ali, (2017). Morphological Identification of Horse Flies (Diptera: Tabanidae) and Estimation of their Seasonal Abundance in Al-Showak District, Gedaref State, Eastern Sudan. IRA-International Journal of Applied Sciences. 6:41-54.

Foil L.D. (1989). Tabanids as vectors of disease agents.Parasitol. Today, 5, 8896.

Luckins AG, (2004). Non-tsetse-transmitted animal trypanosomiasis. In: Maudlin I, et al., eds. the Trypanosomiases. Oxfordshire, UK: CABI, 269-281.

Desquesnes, M., p. Holzmuller, D. H. Lai, A. Dargantes, Z. R. Lun, and S. Jittapa long. (2013). Trypanosoma evansi and Surra: A review and Perspective on Origin, History, Distribution, Taxonomy, Morphology, Host and Pathogenic Effects. BioMed Research International, Vol, 2013 p. 22.

Oldroyd H (1954). The horseflies (Diptera: Tabanidae) of the Ethiopian Region. Volume II. Tabanus and related genera. British Museum (Natural History), London, $X+341 p p$.

Yagi, A. I. (1968). A contribution to the knowledge of the Tabanidae of the Sudan.Ph.D thesis. University of Khartoum, Sudan.

Ratnasingham S, Hebert PDN (2007) BOLD: 
The Barcode of Life Data System. Molecular Ecology Notes 7: 355-364.

Morita S I, Bayless K M, Yeates D K, Wiegmann B M (2016). Molecular phylogeny of the horse flies: a framework for renewing tabanid taxonomy. Systematic Entomology 41: 56-72.

Mugasa C M, Villinger J, Gitau J, Ndungu N, Ciosi M, Masiga D (2018). Morphological re-description and molecular identification of Tabanidae (Diptera) in East Africa. ZooKeys 769: 117-144. https://doi.org/10.3897/ zookeys.769.21144.

Ford, J. (1976). Trypanosomiasis in the Sudan Republic.a report to the Ministry of Overseas Development, 25pp.

SCDSI. (2018). Saudi Central Department of Statistics and Information.

Al-Sharif, A, (1983). Geography of Kingdom. 2nd part: Southwestern of Saudi Arabia, Mareekh, Riyadh.

Al-Sheik, A. A. (2011). Larval Habitat, Ecology, Seasonal Abundance and Victorian Role in Malaria Transmission of Anopheles arabiensis in Jazan Region of Saudi Arabia. Journal of the Egyptian Society of Parasitology, 41 (3): pp. 615-634.

Mihok, S. (2002). The development of a multipurpose trap (the Nzi) for tsetse and other biting flies. Bulletin of Entomological Research 92: 285-403.

Folmer, O., Black, M., Hoeh, W., Lutz, R. \& Vrijenhoek, R. (1994). DNA primers for amplification of mitochondrial cytochrome c oxidase subunit I from diverse metazoan invertebrates. Molecular Marine Biology and Biotechnology, 3, 294-299.

Altschul, S. F., Madden, T. L., Schäffer, A. A., Zhang, J., Zhang, Z., Miller, W. and Lipman, D. J., (1997). Gapped BLAST and PSI-BLAST: a new generation of protein database search programs. Nucleic acids research, 25 (17), pp.3389-3402.

Hall, T. A. (1999). BioEdit: A User- Friendly Biological Sequence Alignment Editor and Analysis Program for Windows 95/98/NT. Nucleic Acids Symposium series, 41, 95-98.

Kumar S., Stecher G., Li M., Knyaz C., and Tamura K. (2018). MEGA X: Molecular Evolutionary Genetics Analysis across computing platforms. Molecular Biology and Evolution 35:1547-1549.

Saitou N. and Nei M. (1987). The neighborjoining method: A new Method for Reconstructing Phylogenetic Trees. Molecular Biology and Evolution 4:406-425.

Felsenstein J. (1985). Confidence limits on phylogenies: An approach using the bootstrap. Evolution 39:783-791.

StjepanKr mar, Davorka K. H., Branimir K. H., (2011). Key to the horse flies fauna of Croatia (Diptera, Tabanidae).Period biol, Vol 113, Suppl 2.

Martin Drake., Alan E. Stubbs (2018). British Soldier flies and their Allies.British Entomological and Natural History Society.Pp.1-14.

Krinsky, W. L. (1976). Animal disease agents transmitted by horse flies and deer flies (Diptera: Tabanidae). Journal of Medical Entomology 13: 225-275.

Barros A. T. M. (2001). Seasonality and relative abundance of Tabanidae (Diptera) captured on horses in the Pantanal, Brazil. Memórias do Instituto Oswaldo Cruz, Rio de Janeiro, 96 (7), 917-923.

Chvála, M., Lyneborg, L. and Moucha, J. (1972). The horse flies of Europe (Diptera, Tabanidae). 500 pp. Entomological Society of Copenhagen, Copenhagen.

Yeates, D. K., Wiegmann, B. M., Courtney, G. W., Meier, R., Lambkin, C. and 
Pape, T. (2007). Phylogeny and systematics of Diptera: two decades of progress and prospects. Zootaxa 1668, 565-590.

Itina, V. I., Noutcha, A. M. E. and Okiwelu, S. N. (2013). Spatial and temporal distribution of tabanids (Diptera: Tabanidae) in Akwalbom State, Nigeria. Research in Zoology 3, 6265.

Baldacchino, F., Desquesnes, M., Mihok, S., Foil, L. D., Duvallet, G. and Jittapalapong, S. (2014). Tabanids: neglected subjects of research, but important vectors of diseases Infection, Genetic and Evolution 28, 596-615.

Rodrigo Ferreira Krüger1 and Tiago KütterKrolow. (2015). Seasonal patterns of horse fly richness and abundance in the Pampa biome of southern Brazil. Journal of Vector Ecology. Vol. 40, no. 2, 364-372.

Suh, S., H. C. Kim, S. Chong, M. Kim and T. Klein. (2015). Seasonal Abundance of
Deer and Horse flies (Diptera: Tabanidae) in the Northern Part of Gyeonggi-do, Republic of Korea. The Korean Journal of Parasitology 53:307-314.

Mikuška, A., S. Krčmar, A. Radović, and T. Mikuška. (2012). The influence of temperature, precipitation and floods on the development of horse fly populations (Tabanidae) in the alluvial habitats of the danube river in Croatia. Polish J. of Ecol. 60: 395-406.

Issimov A, Taylor DB, Zhugunissov $\mathrm{K}$, Kutumbetov L, Zhanabayev A, Kazhgaliyev N, et al., (2020). The combined effects of temperature and relative humidity parameters on the reproduction of Stomoxys species in a laboratory setting.PLoS ONE 15 (12).

Hackenberger, B. K., D. Jarić, and S. Krčmar. (2009). Distribution of Tabanids (Diptera: Tabanidae) along a twosided altitudinal transect. Environ. Ent. 38:1600-1607.

\section{How to cite this article:}

Elobaid, N. I., O. M. Daffalla, S. S. M. Elhasan, E. M. Noureldin, F. F. Ali and Abdalla, M. A. 2021. Morphological, Molecular Identification of Tabanidae (Diptera) and Assessment of their Seasonal Abundance in Southwestern, Saudi Arabia. Int.J.Curr.Microbiol.App.Sci. 10 (04): 485-499. doi: https://doi.org/10.20546/ijcmas.2021.1004.050 